\title{
First results from Project SUNBIRD: Supernovae UNmasked By Infra-Red Detection
}

\author{
Erik C. Kool ${ }^{1,2}$, Stuart D. Ryder ${ }^{2}$, Erkki Kankare ${ }^{3}$, Seppo Mattila ${ }^{4}$ \\ ${ }^{1}$ Dept. of Physics and Astronomy, Macquarie University, Sydney NSW 2109, Australia \\ email: erik.kool@students.mq.edu.au \\ ${ }^{2}$ Australian Astronomical Observatory, Sydney NSW 1670, Australia \\ ${ }^{3}$ Astrophysics Research Centre, Queen's University Belfast, United Kingdom \\ ${ }^{4}$ Tuorla Observatory, University of Turku, Kaarina, Finland
}

\begin{abstract}
A substantial number of core-collapse supernovae (CCSNe) are expected to be hosted by starbursting luminous infrared galaxies (LIRGs). However, so far very few CCSNe have been discovered in LIRGs, most likely as a result of dust extinction and lack of contrast in their typically luminous and complex nuclear regions. We present the first results of Project SUNBIRD (Supernovae UNmasked By InfraRed Detection), where we aim to uncover dust-obscured nuclear supernovae by monitoring over 30 LIRGs, using near-infrared state-of-the-art Laser Guide Star Adaptive Optics (LGSAO) imaging on the Gemini South and Keck telescopes. Such discoveries are vital for determining the fraction of supernovae which will be missed as a result of dust obscuration by current and future optical surveys.
\end{abstract}

Keywords. supernovae: general - galaxies: starburst - infrared: galaxies - instrumentation: adaptive optics - instrumentation: high angular resolution

\section{Summary}

The observed rate of CCSNe has been shown to trail the rate predicted from the cosmic star formation rate by as much as a factor of two (Horiuchi et al. 2011). One of the main suspects for this discrepancy is dust: the light of supernovae gets obscured and traditional optical supernova surveys fail to detect them, in particular in the nuclear regions of their host galaxies. In the dusty star forming LIRGs this effect is most prominent and earlier work by our collaboration showed that most, if not all of the missing supernovae might be accounted for by dust extinction alone (Mattila et al. 2012). However these limits are based on a handful of CCSNe in a single monitored LIRG and thus have large uncertainties.

The aim of Project SUNBIRD is to increase the number of CCSNe found in LIRGs, and in this way improve the constraints on the fraction of CCSNe that are missed in dust obscured nuclear regions. We observe in the near-IR where dust extinction is reduced by a factor of 10 compared to the optical, and make use of telescopes equipped with LGSAO to supply the spatial resolution required to identify nuclear SNe. So far in the first year of SUNBIRD we have discovered three CCSNe, two of which were located within $1 \mathrm{kpc}$ of the nucleus, with extinctions up to 5 magnitudes in $V$ (Kool et al. 2017, in prep).

\section{References}

Horiuchi S. et al. 2011, ApJ, 738, 154

Mattila S. et al. 2012, ApJ, 756, 111 